\title{
Does vocational training encourage continuing professional development?
}

\author{
J. P. Ralph, ' P. E. Mercer, ${ }^{2}$ and H. Bailey, ${ }^{3}$
}

\section{The aim of this study was to compare the continuing professional development of young dentists who had participated in a vocational training scheme with that of young dentists who had not. It was encouraging to find both groups were actively involved in a broad range of continuing educational activities.}

$\mathrm{V}$ ocational training provides a structured introduction to dental practice and it is hoped that the combination of clinical experience and study days will foster a commitment to continuing professional development.

A survey of graduates from Scottish Dental Schools revealed that attendance at postgraduate education sessions amongst young dentists was indeed higher than for the profession as a whole and was associated with increased feelings of competence. ${ }^{1}$ The majority felt that vocational training had encouraged continuing professional development, though just over a quarter disagreed. Very few had obtained further qualifications.

A British Dental Association survey concluded that young dentists were supportive of continuing education but attributed their increased competency and confidence to experience rather than vocational training. ${ }^{2}$ The survey also found that the majority of participants were pleased they had chosen dentistry as a career.

From 1 October 1993 it became mandatory for all newly-qualified dentists who wished to become principals in general dental practice within the NHS to undertake a 1 -year vocational training course. Prior to this, vocational training for dental graduates had been optional. This study reports the findings of a postal survey conducted

${ }^{1}$ J. P. Ralph, Dean of Postgraduate Dental Education, ${ }^{2}$ P. E. Mercer, Research Officer, ${ }^{3} \mathrm{H}$. Bailey, Regional Adviser in General Dental Practice, The Department for NHS Postgraduate Medical \& Dental Education, Willow Terrace Road, University of Leeds, Leeds Correspondence to: Mrs. P.E. Mercer, The

Department for NHS Postgraduate Medical \& Dental Education, Willow Terrace Road, University of Leeds, Leeds LS2 9JT

email:p.mercer@yorkshiredeanery.com

REFEREED PAPER

Received 22.08.00; Accepted 13.02.01

(C) British Dental Journal 2001; 190: 91-96

\section{In brief}

- Vocational dental practitioners (VDPs) and non-vocational dental practitioners (non-VDPs) were actively keeping up-to-date

- The continuing professional development activities of VDPs and non-VDPs were very similar

- Very few in each group had additional qualifications

- Both VDPs and non-VDPS indicated that discussion with colleagues was the most useful medium for keeping up-to-date

- A higher proportion of female VDPs than non-VDPs were principals

towards the end of 1997 which looked at the continuing professional development activity of two groups of young dentists working in general dental practice 7 to 9 years after qualification. The first group chose to enter general dental practice via the vocational training route whilst the second group entered dental practice as associates. A previous paper by the authors reported on the experiences of the same two groups during their first year in practice as vocational trainees and associates. ${ }^{3}$

\section{Method and materials}

The sample

The sample was drawn from dentists who graduated from the University of Leeds Dental School during the years 1988-1990, dentists who graduated elsewhere, but participated in a vocational training scheme in Yorkshire in 1989-1991, and dentists who graduated from other dental schools in 1988-1990 and who were practising in Yorkshire in 1997. Postal questionnaires were sent to the 210 dental graduates selected and at the final count $73 \%$ (154) of the question- naires had been returned and found suitable for inclusion in the study.

For the purpose of analysis the sample was sorted into the following categories:

- Dental graduates not working in the General Dental Services in the UK in 1997

- Dental graduates working in the General Dental Services in 1997 and who had participated in a vocational training scheme (VDPs)

- Dental graduates working in the General Dental Services in 1997 and who had not participated in a vocational scheme (nonVDPs)

\section{The questionnaires}

A questionnaire was designed for graduates who had participated in a vocational training scheme and a second questionnaire for graduates who did not do vocational training. The questionnaires covered three main areas. The first section asked for personal details, eg age, gender and qualifications. The second section sought the opinions and experiences of the newly-qualified dentists during their first year in practice as vocational trainees or associates. The final section asked, in detail, about their continuing professional development. This paper is concerned only with sections 1 and 3 .

\section{Data analysis}

The resultant data were analysed using the statistical package SPSS for Windows.

In order to facilitate further comparison between the two cohorts working in the GDS, ie VDPs and non-VDPs, the Index of Continuing Professional Development (ICPD) was used. ICPD was reported in a study of audit and continuing professional development of general dental practitioners in Yorkshire in $1995^{4}$ in which it was used to summarise the continuing professional development activities of each of the respondents in a range of selected educational activities. In this study it was used to determine the extent to which individual VDPs and non-VDPs were participating in a range of educational activities and the intensity of activity for each group.

\section{Results}

Of the 154 respondents used in the study 
Fig. I Usefulness of sources of information (VDPs, $n=69$ non-VDPs, $n=63$ )

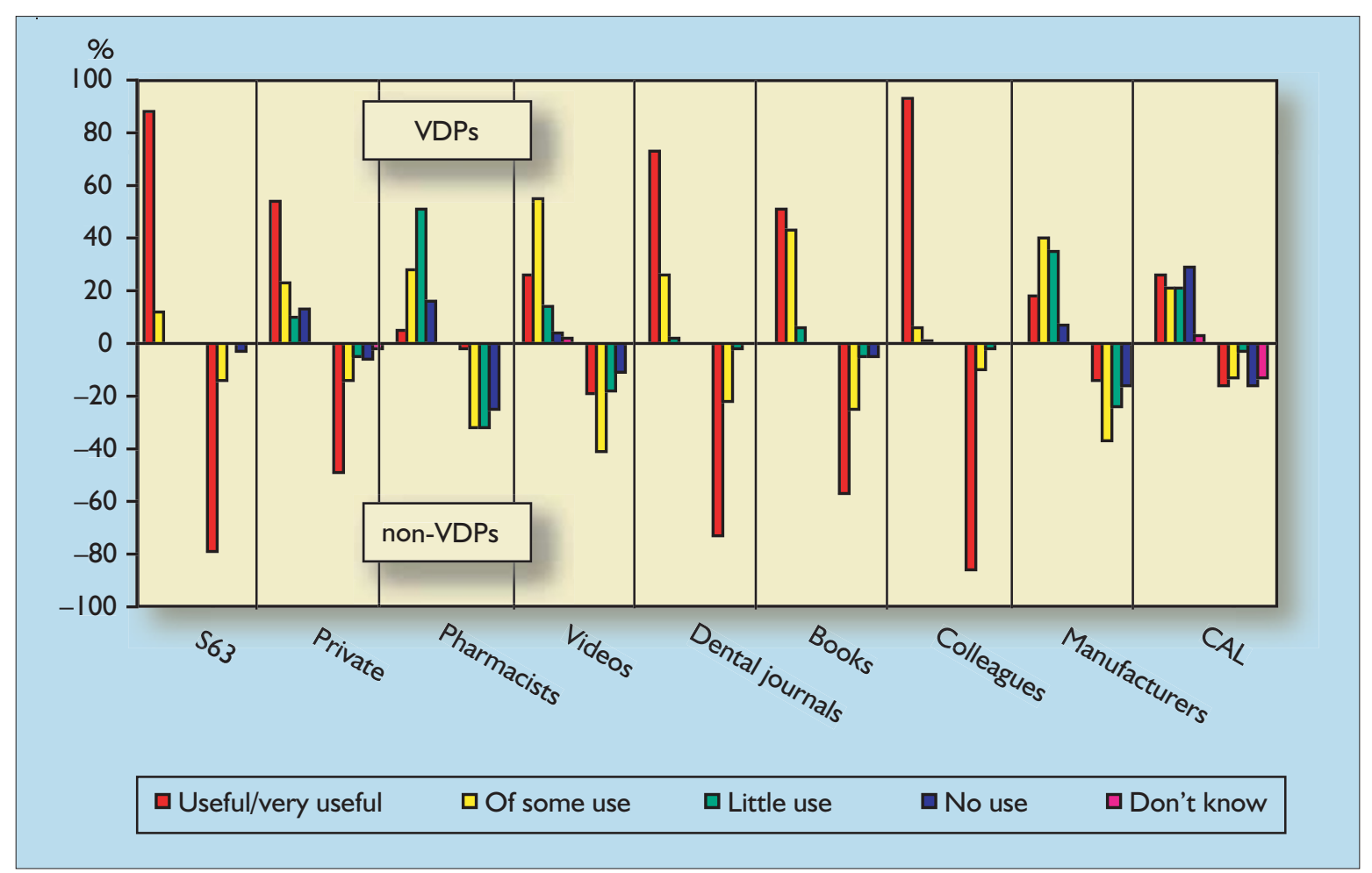

$82(53 \%)$ were male and $72(47 \%)$ were female. This approximates closely to the proportion of male and female graduates from the Leeds Dental School between 1988 and 1990: 51\% male and 49\% female. However, a slightly higher proportion of females undertook vocational training and they accounted for $54 \%$ of the vocational dental practitioner group. More than half of the sample, $57 \%$, had graduated from the Leeds Dental School and the remainder from the various dental schools throughout Britain. The majority of the dentists, $84 \%$, fell into the $30-34$ year age group. During the period the study was carried out 132 of the respondents were working in the General Dental Services.

Dental graduates (VDPs \& non-VDPs) not working in the General Dental Services (UK) in 1997

Twenty-two respondents, 7 male and 15 female, were not working in the General Dental Services (GDS) when the study was carried out. Eleven of these, 5 males and 6 females, were following a career in the Hospital Service and the remaining 2 male respondents were studying medicine. Three female respondents were working full-time in the Community Dental Service (CDS) and another was working part-time in the CDS and part-time in the Hospital Service. A further 3 females were out of the country: 1 was practising dentistry in Australia and another in Portugal whilst the third had taken a break to travel. Only 2 respondents, both females, had taken an extended career break to look after their children. Sixteen had other qualifications and 3 indicated that they intended to seek further qualifications.

Twelve of this group had entered the GDS immediately after graduation, whilst 9 went into the Hospital Service and 1 entered the CDS. Eight participated in a vocational training scheme with 7 commencing vocational training (VT) immediately following graduation. Six VDPs and 3 non-VDPs indicated that their first year in practice had encouraged them to continue with their postgraduate education. Eleven had had a break in their career and 4 indicated that their partner's job had affected their career pathway. As these dentists were not in general dental practice at the time of the survey they were not asked to complete the CPD questionnaire.

Vocational trainees (VDPs) and nonvocational trainees (non-VDPs) working in the GDS in 1997

Sixty-nine respondents $(33$ males, 36 females) had participated in a vocational training scheme and all of these commenced VT immediately following graduation, including one dentist who joined the army. Of the 63 respondents (42 males, 21 females) who did not participate in a vocational training scheme $70 \%$ entered the GDS immediately following graduation, 29\% became House Officers and 1 graduate joined the Royal Army Dental Corps. At the time of the study $96 \%$ VDPs and $94 \%$ nonVDPs were working full-time in the General Dental Services. Two VDPs were working part-time in the CDS and part-time as general dental practitioners and 1 was working as a dental officer with the Army. Three non-VDPs were sharing their time between the Hospital Service and the GDS and 1 between the CDS and the GDS.

Seventeen per cent of VDPs and 19\% of non-VDPs had additional qualifications 


\begin{tabular}{|c|c|c|c|c|c|c|c|c|}
\hline \multirow[t]{2}{*}{ National association } & \multicolumn{2}{|c|}{ VDPs } & \multicolumn{2}{|c|}{ non-VDPs } & \multirow[t]{2}{*}{ Local associations } & \multicolumn{2}{|c|}{ VDPs } & non-VDPs \\
\hline & $n$ & $\%$ & $n$ & $\%$ & & $\mathbf{n}$ & $\%$ & $n \quad \%$ \\
\hline British Dental Association & 38 & 88 & 31 & 70 & LDC & 5 & & 3 \\
\hline $\mathrm{FGDP}(\mathrm{UK})$ & 5 & & 2 & & IPG & 3 & & 3 \\
\hline GDPA & 2 & & 5 & & Local dental societies & 5 & & 7 \\
\hline Specialist societies & 4 & & 4 & & & & & \\
\hline
\end{tabular}

whilst 32\% and 39\% respectively said they intended to obtain further qualifications and $38 \%$ and $30 \%$ indicated that they might sit for further qualifications.

Thirty-five per cent of VDPs and 27\% of non-VDPs indicated that they had had a break in their career. Nineteen VDPs had breaks for maternity leave, 7 used their career breaks to travel and 4 had breaks to study with one qualifying as a solicitor, one gaining a diploma in Law, and another undertaking a self-funded masters degree. Seven of the non-VDPs had breaks for maternity leave and 6 had breaks to travel or work abroad. One non-VDP had a 2-year break to study for the MSc in Restorative Dentistry and 2 had breaks because of illness. The majority of breaks lasted 3-6 months but two respondents each had had breaks of 2 years.

When asked if their partner's job had affected their career pathway $22 \%$ of each group indicated that it had. The most frequently quoted reason was confining their job search to the same area as their partner. Seven non-VDPs indicated that they were constrained by their partners' jobs and 5 were supporting partners who were undertaking further studies. One dentist said his wife had influenced his decision-making with regard to his career and another that her choice of jobs was limited because of the distance to travel.

Thirty-eight per cent of VDPs and $44 \%$ of non-VDPs were principals in their prac- tices. In the VDP group 25\% of females were principals compared with $52 \%$ of males, and in the non-VDP group 19\% of the females were principals compared with 57 $\%$ of males. The majority of dentists, $70 \%$ of VDPs and $66 \%$ of non-VDPs worked in practices with 2 or more colleagues. A further $13 \%$ and $24 \%$ respectively worked with another dentist and $17 \%$ and $10 \%$ worked alone. Fifty-nine per cent of VDPs and 57\% of non-VDPs had a computer in their practice, $49 \%$ and $56 \%$ a computer in their home and $32 \%$ and $33 \%$ computers at home and in the practice. Thirty-three per cent of VDPs and $31 \%$ of non-VDPs considered their knowledge of computers to be average but $13 \%$ and $21 \%$ indicated they had above average or excellent knowledge. Skills in using software were along similar lines. Thirty-three per cent of VDPs and $30 \%$ of non-VDPs had used CAL programs with the majority using the dental programs provided by the Department of Health.

Respondents were asked if they had any regular professional commitments other than to their practice and $17 \%$ of VDPs and $25 \%$ of non-VDPs indicated that they had other commitments. Of the 12 VDPs with other commitments: 4 were involved in hospital attachments, 4 in vocational training, 2 in postgraduate teaching and 1 in undergraduate teaching. Other commitments included membership of a local dental committee, dental adviser on a complaints committee and working part-time as a community practitioner. Of the 16 nonVDPs: 8 were involved in hospital attachments, 3 in vocational training, 3 in postgraduate teaching, 3 in undergraduate teaching and 2 had other commitments. Thirteen per cent of VDPs and $22 \%$ of nonVDPs indicated that they had specialty interests. Three VDPs specialised in Restorative Dentistry, 2 in Orthodontics and 2 in Oral Surgery. Other specialities included endodontics, paedodontics, intravenous sedation and occlusion. Six nonVDPs specialised in oral surgery, 2 in orthodontics, 2 in endodontics, 1 in periodontology and 1 in restorative dentistry.

Many of the respondents were involved in a number of continuing professional development activities. These included membership of professional associations, attending courses, participating in study groups and peer review groups, carrying out audit in the practice, reading journals and using the Self-Assessment Manual and Standards (SAMS) published by the faculty of General Dental Practitioners (UK). ${ }^{5}$

Sixty-two per cent of VDPs and $74 \%$ of non-VDPs were members of a national or local professional association (Table 1). Fifteen VDPs and 8 non-VDPs were members of two associations and 4 from each group held membership with three associations. Eighty-eight per cent of VDPs and 70\% of non-VDPs belonging to professional associations were members of the BDA. Five VDPs and 2 non-VDPs were members of the 


\section{$\%$ VDPs}

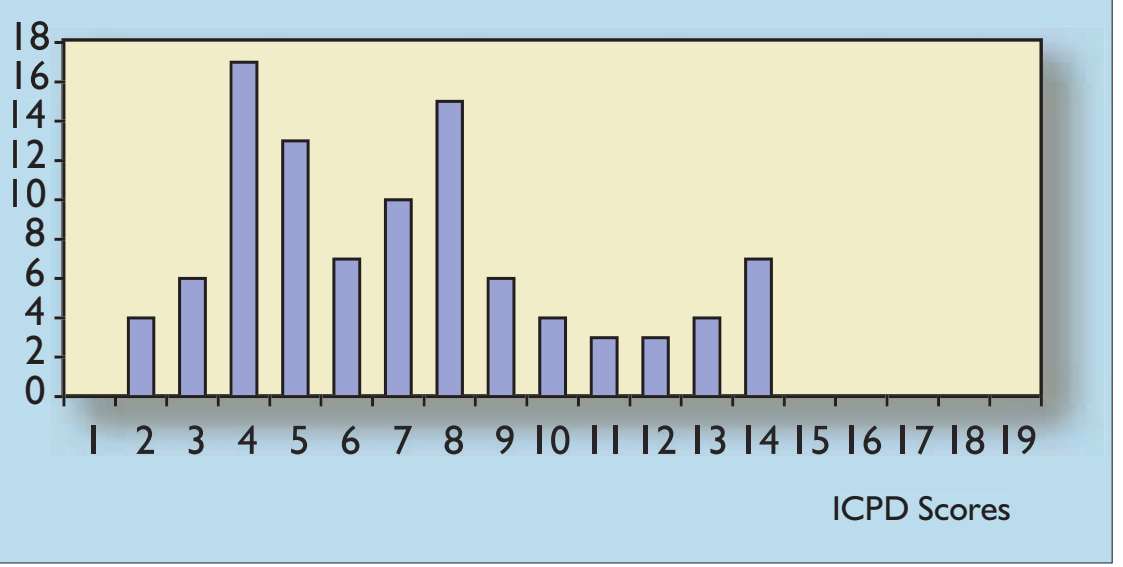

Fig. 2 ICPD scores for VDPs

FGDP (UK) and 5 non-VDPs held GDPA membership. Just over half of the VDPs and just under half of the non-VDPs said they attended professional association meetings regularly. At the other end of the scale 15\% and $34 \%$ respectively said they never attended at all. Seven respondents from each group were or had been officers in their associations.

Nearly half of the respondents in each group had participated in peer review ie $46 \%$ of VDPs and $43 \%$ of non-VDPs. Ten VDPs and 8 non-VDPs belonged to study groups. Just under half of the VDP group and just over half of the non-VDP group who had participated in peer review thought the major advantage in taking part in peer review was the sharing of knowledge with peers.

A further $22 \%$ and $26 \%$ respectively considered meeting other dentists a major advantage. Seventy-five per cent of VDPs and $41 \%$ of non-VDPs who responded to the question on the disadvantages of peer review felt there were no disadvantages associated with it.
Only $10 \%$ of VDPs and $14 \%$ of non-VDPs indicated that they had a good or very good knowledge of audit with $70 \%$ and $67 \%$ respectively indicating that their knowledge was very limited. However, 13 practitioners in each group indicated they had used audit in their practice with 7 VDPs and 8 nonVDPs receiving Department of Health funding for audit projects.

Two VDPs and 5 non-VDPs had attempted to undertake audit in their practice but had not been successful. Six VDPs and 8 non-VDPs had attended an educational activity on audit but $35 \%$ and $30 \%$ respectively felt they needed to learn more about audit with the majority wanting to know what audit is and how to implement it. Less than half of each group, $43 \%$ and $48 \%$ gave their opinions on the advantages and disadvantages of audit. Of these, the majority thought its main advantage was the monitoring and adjusting of standards and its main disadvantage was the amount of time involved in carrying out audit.

SAMS was used by $49 \%$ of VDPs and $43 \%$ of non-VDPs with the vast majority of those who had used SAMS indicating that they had found it useful and that they had used it as a guide to setting standards. Fifty-six per cent of VDPs and $41 \%$ of non-VDPs used it for discussion purposes, $44 \%$ and $41 \%$ for teaching and $32 \%$ and $26 \%$ used it in peer

\section{Table 2 Index of Continuing Professional Development (ICPD) components}

\section{Variable}

Professional association participation

Section 63 course attender

Private course attender

Journal consultation

Used audit

Used SAMS

Peer review participation

Study group membership

Used CAL

Additional qualifications

Other commitments

\section{Levels}

Non-member (0), never/rarely (I), sometimes (2), regular (3)

Non-attender (0), I-2 courses (I), 3-4 courses (2), 5+ courses (3)

Non-attender (0), I-2 courses (I), 3-4 courses (2), 5+ courses (3)

None (0), I-2 journals (I), 3-4 journals (2), $5+$ journals (3)

No (0) Yes (I)

No (0) Yes (I)

No (0) Yes (I)

No (0) Yes (I)

No (0) Yes (I)

No (0) Yes (I)

No (0) Yes (I) 
review meetings. The major advantage to using SAMS as indicated by both groups was its usefulness as a guide to setting standards. The main disadvantage of SAMS was that it was too idealistic and some dentists felt it was limited and over simplified.

The journals most frequently found in the practice were Dental Practice ( $87 \%$ \& $86 \%$ ), the British Dental Journal (86\% \& 73\%), The Dentist (80\% \& 87\%), The Probe (80\% \& $73 \%)$, Dental Update (65\% \& 52\%). The British Dental Journal was the most regularly read (65\% \& 46\%) followed by Dental Practice (57\% \& 43\%), The Probe (46\%), Dental Update (45\% \& 43\%) and The Dentist (42\% \& 46\%). A small group of practitioners took Quintessence International and $19 \%$ of VDPs and $16 \%$ of nonVDPs had other journals in their practice.

Ninety per cent of VDPs and $89 \%$ of nonVDPS had attended at least one MADEL funded (Section 63) course in the previous academic year and $49 \%$ and $54 \%$ had attended at least one private course.

Twenty-three per cent and $21 \%$ had attended five or more Section 63 courses and $6 \%$ of each group had attended five or more private courses. Only $10 \%$ in each group had not attended any course in the previous year. Those dental practitioners who were not frequent attenders at Section 63 courses were asked to indicate from a list of reasons for non-attendance which were of most importance. Distance to travel, timing of courses and pressure of work were the main reasons given by both groups for nonattendance.

Respondents were asked to rate how useful they found a range of media which could be used by dental practitioners to update their knowledge and skills (Figure 1). Discussion with colleagues was the most highly rated medium (93\% \& 86\%) closely followed by Section 63 courses ( $88 \%$ \& 79\%) and the dental journals (73\% \& 73\%).

When respondents were asked if vocational training or first year in practice had encouraged them to continue with their professional development $74 \%$ of VDPs and $54 \%$ of non-VDPs answered positively.

Seventy-two per cent of VDPs and 92\% of non-VDPs replied to the question which asked about methods used to keep

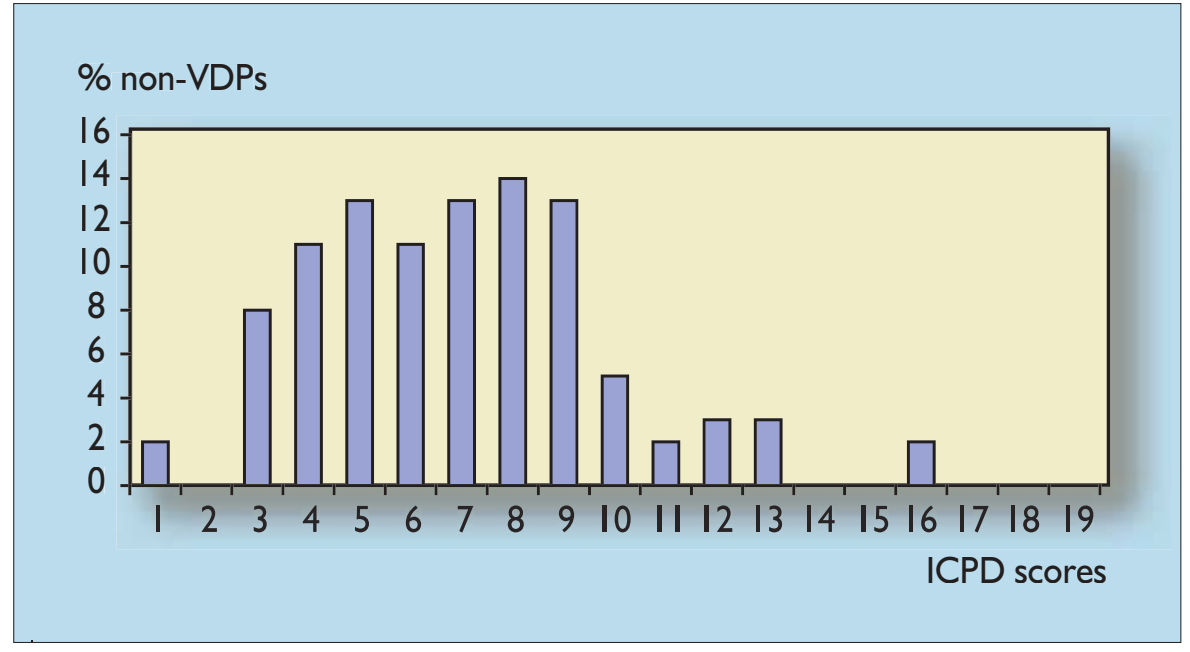

\section{Fig. 3 ICPD scores for non-VDPs}

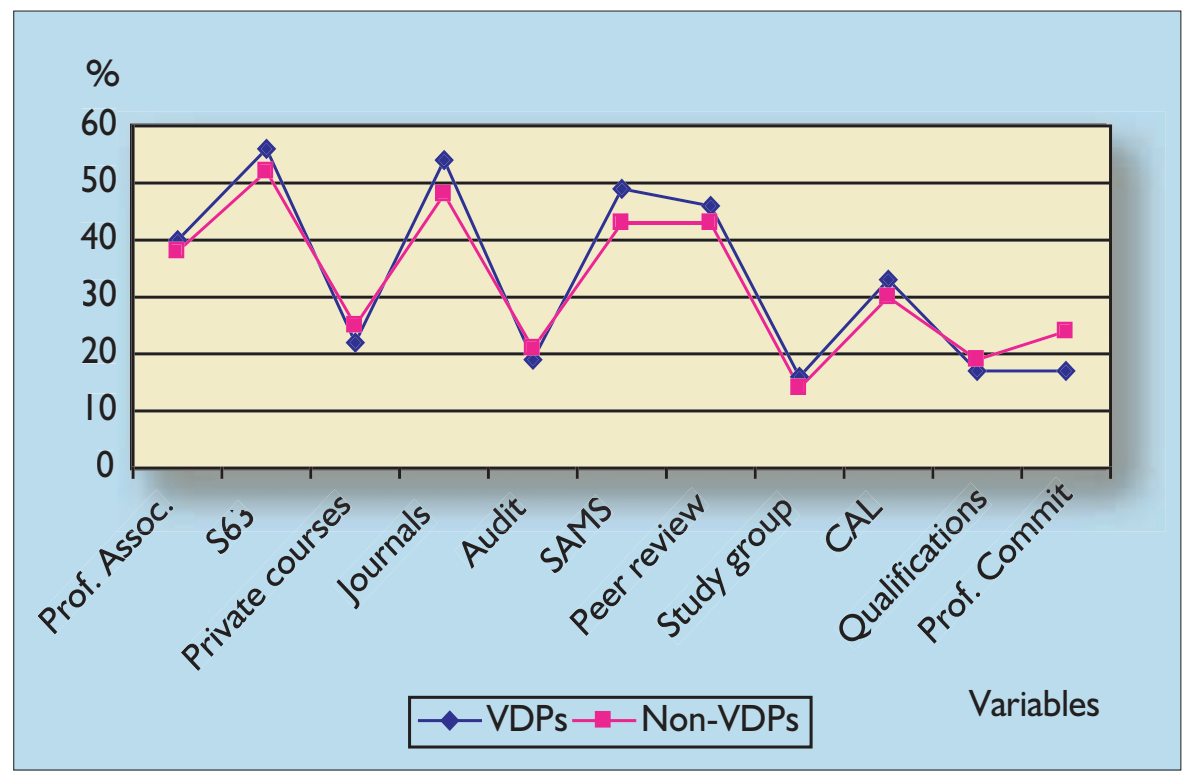

Fig. 4 Total group scores for VDPs and non-VDPs for each variable

up to date. Sixty-six per cent and $88 \%$ respectively said they attended courses, $22 \% \& 17 \%$ participated in postgraduate study, $18 \% \& 12 \%$ used peer review and 2 VDPs and 4 non-VDPs had become trainers themselves.

The respondents were very positive about their choice of career. Replying to the question as to whether or not dentistry had met their personal and professional needs, over $80 \%$ in each group said they were satisfied or very satisfied with their chosen career.

\section{An Index of Continuing Professional Development (ICPD)}

The range of continuing educational activities on which information was obtained in the survey was listed and points awarded for degrees of activity in each area. For example, non-attendance at Section 63 courses was allocated 0 points and regular attendance 3 points (Table 2). The resulting index scores run from 0 to 19 . The index is simple, attaching equal weighting to each of its components. It is not uni- 
dimensional or strictly additive, it comprises a number of dimensions, but it provides an elementary way to summarise the continuing professional development activities of each dentist.

Figure 2 shows the distribution of VDPs on the Index. The median score is 7 . Fortysix per cent of VDPs scored below 7 and $42 \%$ scored between 8 and 14 points. Nobody scored above 14 points.

Figure 3 shows the distribution of nonVDPs on the index scale. The median score is 7. Forty-five per cent of non-VDPs scored below 7 and 42\% scored between 8 and 16 points. Nobody scored above 16 points

Figure 4 illustrates the scores for the intensity of activity achieved by each group for the individual variables. The scores achieved by each group for the individual variables are almost identical. Non-VDPs scored slightly higher in professional commitments outside the practice and VDPs in use of SAMS.

\section{Discussion}

The group of dentists who were not working in general dental practice had completed only the first section of the questionnaire. Their pattern of career breaks was similar to that of the other groups. A higher proportion had obtained further qualifications but this was not surprising as many of them were working in salaried services where career progression is strongly dependent on additional qualifications.

Of the two cohorts of dental graduates who had followed careers in the general dental services, both the VDP and non-VDP groups were actively keeping up to date. There was also a remarkably similar pattern in the continuing professional activities of the two groups.

Both groups rated discussion with colleagues as the most useful medium for updating their knowledge. This was followed closely by Section 63 courses and then dental journals. They demonstrated a similar pattern of attendance at Section 63 Courses, private courses and membership of national and local professional organisations. Both groups used a number of other methods of keeping up to date but in similar proportions. These included SAMS, Clinical Audit and Peer Review. Taking the two groups as a whole, it was disappointing to find that clinical audit had not been used more widely. It was nevertheless encouraging to find that these young practitioners had a greater knowledge of and were much more actively involved in audit than the respondents to a previous survey carried out in Yorkshire in 1995. ${ }^{4}$ Positive findings in regard to quality assurance activities were the participation of nearly half of each group in peer review and the use of SAMS. On a less positive note only a small proportion of each cohort belonged to study groups and very few had additional qualifications, $17 \%$ of VDPs and $19 \%$ of non-VDPs. Nevertheless, a sizeable proportion, $32 \%$ and $39 \%$ respectively, indicated that they intended to seek further qualifications.

It was interesting to note, across both groups, that availability of computers in the practice and at home had increased since previous studies and that familiarity and confidence in their use had also improved. Both groups were familiar with and had made use of CAL programs.

When the ICPD was used as a comparator, this further reinforced the similarities in the continuing professional development of the two groups; they shared a median score of 7. Individuals in the nonVDP group recorded the highest and also lowest scores, while a slightly higher proportion of VDPs recorded scores of 10 or more. The intensity of activity achieved by each group for the individual variables was almost identical.
Taking the two groups as a whole it was interesting to note that less than $3 \%$ of female practitioners had taken time out to become full-time mothers and that a reasonable number had become principals in practice, rather more VDPs than nonVDPs, 25\%:19\%.

\section{Conclusion}

It was reassuring to discover that those young dentists who had undertaken vocational training had maintained an interest in their continuing professional development and were using a broad range of educational opportunities for this purpose. It was equally encouraging to find that the group of young dentists who had not undertaken vocational training, and who perceived that the VDP group might be more actively involved in CPD were themselves engaging in a broadly similar range and volume of postgraduate educational activity. This must augur well for the future of the dental profession, particularly in view of the recent introduction of the General Dental Council's re-certification scheme 'Lifelong Learning - Taking Dentistry Forward'.

The authors are most grateful to the general dental practitioners who participated in this study.

1 Baldwin P J, Dodd M, Rennie J S. Postgraduate dental education and the 'new' graduate. $\mathrm{Br}$ Dent J 1998; 185: 591-594.

2 Survey of Young Dentists 1998 British Dental Association Research Unit.

3 Ralph J P, Mercer P E, Bailey H. A comparison of the experiences of newly qualified dentists and vocational dental practitioners during their first year of general dental practice. $\mathrm{Br}$ Dent J 2000; 189: 101-106.

4 Mercer P E, Long A F, Ralph J P, Bailey H. Audit Activity and uptake of postgraduate dental education among general dental practitioners in Yorkshire. Br Dent J 1998; 184: 138-142.

5 Self Assessment Manual and Standards 1991. Advisory Board in General Dental Practice, Faculty of Dental Surgery, Royal College of Surgeons of England. 